\title{
High-resolution X-ray spectroscopy of Procyon by Chandra and XMM-Newton
}

\author{
A. J. J. Raassen ${ }^{1,2}$, R. Mewe ${ }^{1}$, M. Audard ${ }^{3}$, M. Güdel ${ }^{3}$, E. Behar ${ }^{4}$, J. S. Kaastra ${ }^{1}$, R. L. J. van der Meer ${ }^{1}$, \\ C. R. Foley ${ }^{5}$, and J.-U. Ness ${ }^{6}$
}

\begin{abstract}
1 SRON National Institute for Space Research, Sorbonnelaan 2, 3584 CA Utrecht, The Netherlands
2 Astronomical Institute "Anton Pannekoek", Kruislaan 403, 1098 SJ Amsterdam, The Netherlands

3 Paul Scherrer Institut, Würenlingen \& Villigen, 5232 Villigen PSI, Switzerland

4 Columbia Astrophysics Laboratory, Columbia University, New York, NY 10027, USA

5 Mullard Space Science Laboratory, University College London, Surrey, RH5 6NT, UK

${ }^{6}$ Universität Hamburg, Gojenbergsweg 112, 21029 Hamburg, Germany
\end{abstract}

Received 27 August 2001 / Accepted 5 April 2002

\begin{abstract}
We report the analysis of the high-resolution soft X-ray spectrum of the nearby F-type star Procyon in the wavelength range from 5 to $175 \AA$ obtained with the Low Energy Transmission Grating Spectrometer (LETGS) on board Chandra and with the Reflection Grating Spectrometers (RGS) and the EPIC-MOS CCD spectrometers on board XMM-Newton. Line fluxes have been measured separately for the RGS and LETGS. Spectra have been fitted globally to obtain self-consistent temperatures, emission measures, and abundances. The total volume emission measure is $\sim 4.1 \times 10^{50} \mathrm{~cm}^{-3}$ with a peak between 1 and $3 \mathrm{MK}$. No indications for a dominant hot component $(T \gtrsim 4 \mathrm{MK})$ were found. We present additional evidence for the lack of a solar-type FIP-effect, confirming earlier EUVE results.
\end{abstract}

Key words. stars: individual: Procyon, $\alpha$ Canis Minoris - stars: coronae - stars: late-type - stars: activity X-rays: stars

\section{Introduction}

Magnetized hot outer atmospheres (coronae) are ubiquitous in cool stars (spectral classes F-M). The particular example of the solar corona has revealed rich details on coronal structures, thermal stratification, abundance patterns, and the physics of heating and mass motion. Nevertheless, in many other stars coronal phenomena not common to the Sun are regularly observed, such as persistent high-density $\left(n_{\mathrm{e}}>10^{10} \mathrm{~cm}^{-3}\right)$ coronal plasmas, persistent very hot gas $(T>10 \mathrm{MK})$, or abundances at variance with solar values (Bowyer et al. 2000). The Sun's relatively modest magnetic activity is representative for a particular evolutionary state of a $1 M_{\odot}$ star (Güdel et al. 1997), while most stellar objects regularly observed by X-ray satellites belong either to the more abundant lowmass classes with some exceptional magnetic activity ( $\mathrm{M}$ dwarfs and young $\mathrm{K}$ dwarfs) or to tidally coupled binary systems with strongly enhanced magnetic dynamos (RS CVn or Algol-type binaries).

High-resolution X-ray spectroscopy of such stellar systems now available from Chandra and XMM-Newton (Brinkman et al. 2000, 2001) has revealed coronal

Send offprint requests to: A. J. J. Raassen,

e-mail: a.j.j.raassen@sron.nl features clearly at variance with solar phenomena. However, to translate solar knowledge to stellar environments, it is important to study stars that are relatively similar to the Sun. Given the low X-ray luminosity of such stars, there are only a few in the solar neighborhood accessible to high-resolution X-ray spectroscopy. We present here a detailed analysis of the X-ray spectrum of Procyon, a nearby bright X-ray source with a coronal plasma of about 1-3 MK, exhibiting a cooler X-ray spectrum than magnetically active stars that have predominantly been studied so far with XMM-Newton and Chandra (Audard et al. 2001a,b; Güdel et al. 2001a,b; Mewe et al. 2001). The late-type (F5 IV-V) optically bright $\left(m_{\mathrm{v}}=0.34\right)$ star Procyon (with a faint white dwarf companion) at a distance of $3.5 \mathrm{pc}$ has a line-rich coronal spectrum in the X-ray region. The mass of Procyon is $1.75 M_{\odot}$ and its radius $2.1 R_{\odot}$ (Irwin et al. 1992). The high-resolution spectrum of Procyon has been studied earlier using EUVE (Drake et al. 1995; Schrijver et al. 1995; Schmitt et al. 1996) and by means of the LETGS on board Chandra (Ness et al. 2001). Ness et al. focussed their efforts on the density-sensitive and temperature-sensitive lines of $\mathrm{C} \mathrm{V}$, N VI, and O VII only. Here we present an extended investigation of the LETGS spectrum covering the total spectral range from $5-175 \AA$ together with the analysis of the 

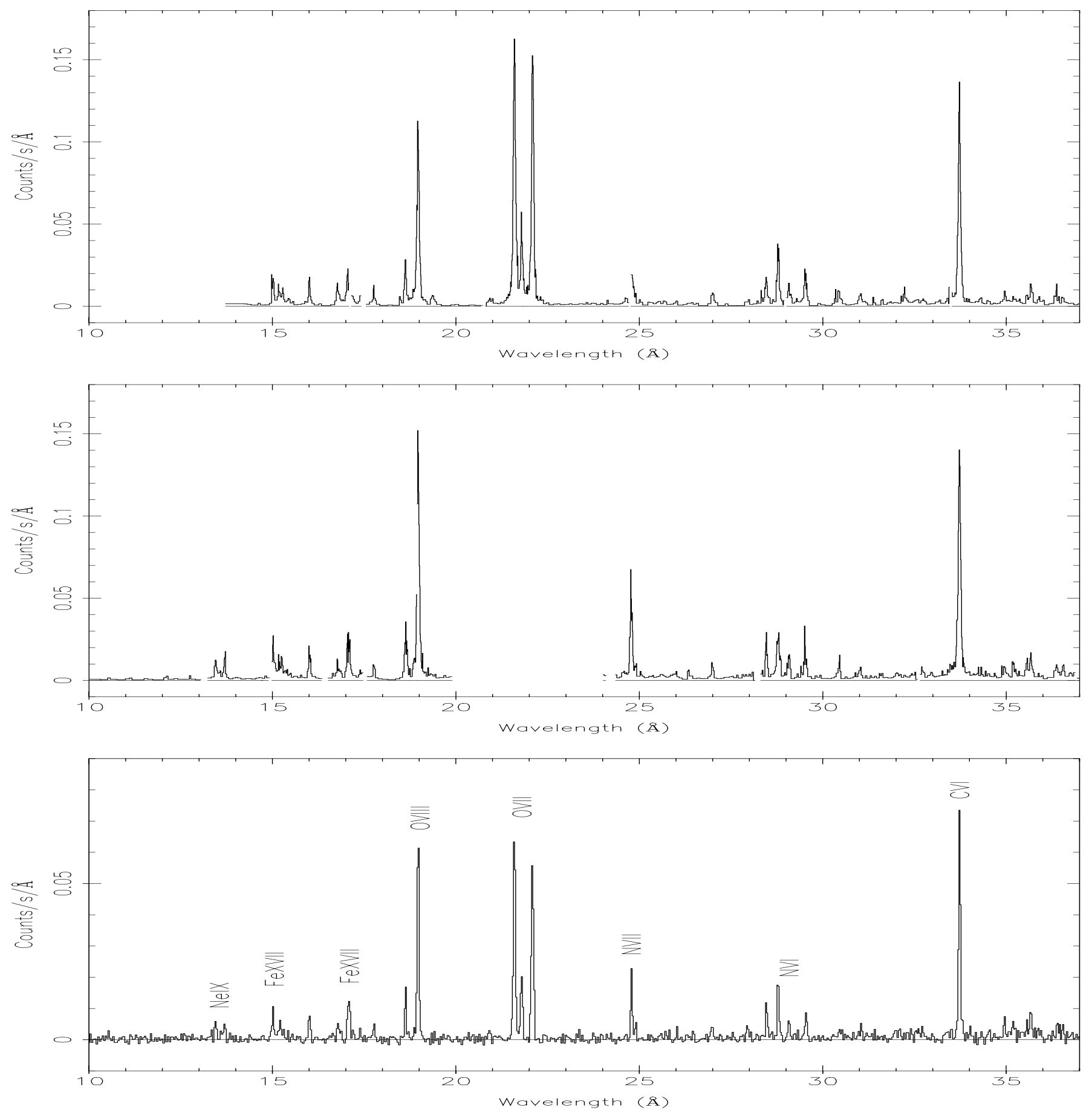

Fig. 1. From top to bottom the spectra of Procyon observed by RGS1, RGS2 and LETGS in the wavelength region from 10 to $37 \AA$.

RGS spectra from 5-37 A. In Sect. 2 we describe the observations. Section 3 (Analysis) is divided into a part on global fitting (3.2) based on the total spectrum and a part that contains consistency checks based on individual line measurements (3.3).

\section{Observations}

The spectrum of Procyon was obtained during $70.4 \mathrm{ksec}$ (on November 8, 1999) by the High Resolution Camera
(HRC-S) and the LETGS on board Chandra. The HRC-S contains three flat detectors, each $10 \mathrm{~cm}$ long. LETGS consists of 180 grating modules. The LETGS spectrum covers the range from 5 to $175 \AA$. The LETGS spectra were summed over the +1 and -1 orders and contain also the higher orders. The higher orders are fitted in the model calculations, but can be neglected for Procyon. The curve of the effective area as a function of wavelength is complicated because of the presence of absorption edges (e.g., around $42 \AA$ ) and gaps between $62-65 \AA$ and $52-56 \AA$ 

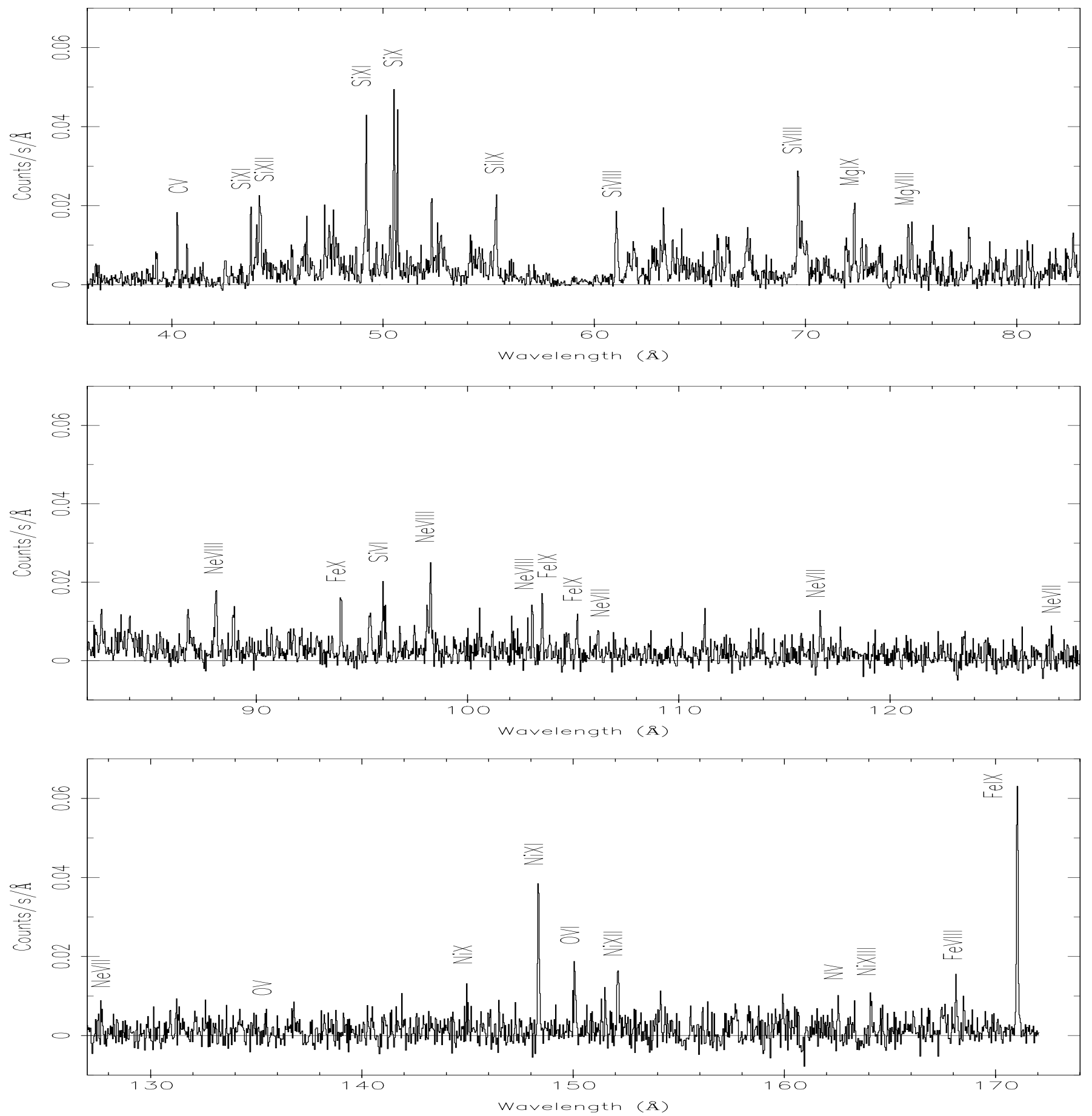

Fig. 2. The Procyon spectrum observed by LETGS in the region $36-174 \AA$.

( +1 and -1 order, respectively) due to the gaps between the detector plates. We use the SRON values (based on calibration by van der Meer et al. 2002), which agree within about $5-10 \%$ with the CXC values, as given in the Chandra LETGS Calibration Review of 31 Oct. $2001^{1}$. The wavelength resolution is $\Delta \lambda \sim 0.06 \AA(F W H M)$. The wavelength uncertainty in the calibration is a few $\mathrm{m} \AA$ below $30 \AA$ and about $0.020 \AA$ in the region above $30 \AA$.

\footnotetext{
${ }^{1}$ http://cxc.harvard.edu/cal/Links/Letg/User/Review_ 311001/eff_area.html
}

The spectra are background subtracted. The statistical errors in the line fluxes include errors from the background. For further instrumental details see also Brinkman et al. (2000).

Later (on October 22, 2000), the spectrum of Procyon was observed by XMM-Newton using the RGS and EPIC-MOS. The total observing time was $\approx 107 \mathrm{ksec}$; however, due to large solar flare activity at the end of the observation, we removed $16.7 \mathrm{ksec}$ of data, leaving a total of $90.5 \mathrm{ksec}$ of "good" data. In XMM-Newton three telescopes focus X-rays onto three EPIC cameras (two MOS 
Table 1. Wavelengths and fluxes for RGS1, RGS2, and LETGS, together with the line identifications from MEKAL and KELLY.

\begin{tabular}{|c|c|c|c|c|c|c|c|c|c|}
\hline \multicolumn{2}{|c|}{ RGS1 } & \multicolumn{2}{|c|}{ RGS2 } & \multicolumn{2}{|c|}{ LETGS } & \multicolumn{4}{|c|}{ Line identifications $^{a}$} \\
\hline$\lambda(\AA)$ & flux $^{b}$ & $\lambda(\AA)$ & flux $^{b}$ & $\lambda(\AA)$ & flux $^{b}$ & MEKAL & flux $^{c}$ & KELLY & Ion \\
\hline gap & - & $13.454(9)$ & $0.17(4)$ & $13.450(13)$ & $0.14(5)$ & 13.448 & 0.14 & 13.447 & Ne IX \\
\hline gap & - & $13.701(6)$ & $0.17(4)$ & $13.690(15)$ & $0.12(5)$ & 13.700 & 0.10 & 13.700 & $\mathrm{Ne} I X$ \\
\hline $15.018(7)$ & $0.20(5)$ & $15.024(9)$ & $0.26(4)$ & $15.015(18)$ & $0.21(6)$ & 15.014 & 0.16 & 15.013 & $\mathrm{Fe} \mathrm{XVII}^{d}$ \\
\hline $15.176(13)$ & $0.12(3)$ & $15.161(21)$ & $0.08(3)$ & - & - & 15.175 & 0.03 & 15.176 & O VIII \\
\hline- & - & - & - & $15.207(28)$ & $0.13(5)$ & - & 0.09 & - & Fe XVII ${ }^{e}$ \\
\hline $15.281(12)$ & $0.11(3)$ & $15.258(12)$ & $0.09(5)$ & - & - & 15.265 & 0.04 & 15.260 & Fe XVII \\
\hline $16.008(3)$ & $0.17(3)$ & $16.013(9)$ & $0.20(5)$ & $16.008(9)$ & $0.18(5)$ & 16.003 & 0.15 & 16.007 & O VIII ${ }^{f}$ \\
\hline $16.776(9)$ & $0.16(3)$ & $16.776(15)$ & $0.12(4)$ & $16.790(14)$ & $0.13(5)$ & 16.780 & 0.10 & 16.775 & Fe XVII \\
\hline $17.047(9)$ & $0.23(4)$ & $17.044(14)$ & $0.19(5)$ & $17.054(12)$ & $0.22(9)$ & 17.055 & 0.12 & 17.051 & Fe XVII \\
\hline- & - & $17.099(12)$ & $0.25(5)$ & $17.102(9)$ & $0.29(9)$ & 17.100 & 0.10 & 17.100 & Fe XVII \\
\hline $17.402(12)$ & $0.07(3)$ & $17.402(28)$ & $0.06(3)$ & $17.396(17)$ & $0.08(5)$ & 17.380 & 0.04 & 17.396 & O VII \\
\hline $17.765(9)$ & $0.10(3)$ & $17.772(9)$ & $0.11(4)$ & $17.769(13)$ & $0.14(5)$ & 17.770 & 0.12 & 17.768 & O VII \\
\hline $18.624(4)$ & $0.30(4)$ & $18.637(6)$ & $0.37(5)$ & $18.629(5)$ & $0.39(8)$ & 18.628 & 0.39 & 18.627 & O VII \\
\hline $18.970(2)$ & $1.61(8)$ & $18.975(2)$ & $1.78(11)$ & $18.972(2)$ & $1.83(15)$ & 18.973 & 1.93 & 18.969 & O VIII \\
\hline $20.918(27)$ & $0.04(3)$ & gap & - & $20.905(22)$ & $0.14(7)$ & 20.910 & 0.06 & 20.910 & N VII \\
\hline $21.596(2)$ & $2.36(11)$ & gap & - & $21.597(2)$ & $3.01(25)$ & 21.602 & 3.35 & 21.602 & O VII \\
\hline $21.797(4)$ & $0.53(6)$ & gap & - & $21.792(5)$ & $0.90(14)$ & 21.800 & 0.80 & 21.804 & O VII \\
\hline $22.098(2)$ & $2.20(11)$ & gap & - & $22.089(2)$ & $2.57(23)$ & 22.100 & 2.33 & 22.101 & O VII \\
\hline - & - & $24.780(3)$ & $0.88(7)$ & $24.790(4)$ & $0.80(14)$ & 24.781 & 0.76 & 24.781 & N VII \\
\hline - & - & $24.907(22)$ & $0.08(4)$ & $24.906(11)$ & $0.18(9)$ & 24.900 & 0.08 & 24.898 & $\mathrm{~N}$ VI \\
\hline $27.001(11)$ & $0.16(4)$ & $26.994(12)$ & $0.14(4)$ & $26.979(19)$ & $0.18(9)$ & 26.990 & 0.08 & 26.990 & C VI \\
\hline $28.460(7)$ & $0.30(5)$ & $28.465(6)$ & $0.39(6)$ & $28.470(6)$ & $0.49(12)$ & 28.470 & 0.39 & 28.466 & C VI \\
\hline $28.785(4)$ & $0.69(9)$ & $28.775(6)$ & $0.71(8)$ & $28.785(5)$ & $0.88(15)$ & 28.790 & 0.77 & 28.787 & N VI \\
\hline $29.078(9)$ & $0.24(5)$ & $29.084(9)$ & $0.29(7)$ & $29.082(12)$ & $0.29(10)$ & 29.090 & 0.32 & 29.084 & N VI \\
\hline $29.524(6)$ & $0.43(6)$ & $29.520(8)$ & $0.38(6)$ & $29.546(11)$ & $0.43(13)$ & 29.530 & 0.41 & 29.534 & $\mathrm{~N}$ VI \\
\hline $30.445(12)$ & $0.20(5)$ & $30.446(13)$ & $0.22(5)$ & $30.450(25)$ & $0.18(11)$ & 30.448 & 0.22 & 30.448 & $\mathrm{Ca}$ XI \\
\hline $31.027(16)$ & $0.18(5)$ & $31.021(12)$ & $0.15(5)$ & $31.054(15)$ & $0.22(10)$ & 31.015 & 0.19 & 31.015 & Si XII \\
\hline- & - & $33.490(26)$ & $0.15(7)$ & $33.510(18)$ & $0.17(10)$ & - & - & - & - \\
\hline $33.724(2)$ & $3.49(17)$ & $33.726(2)$ & $4.15(30)$ & $33.731(2)$ & $4.02(32)$ & 33.736 & 4.64 & 33.736 & C VI \\
\hline $34.967(12)$ & $0.19(6)$ & $34.962(15)$ & $0.17(7)$ & $34.959(15)$ & $0.27(17)$ & 34.970 & 0.22 & 34.973 & $\mathrm{C} \mathrm{V}$ \\
\hline $35.198(30)$ & $0.13(6)$ & $35.193(12)$ & $0.27(8)$ & $35.188(18)$ & $0.29(15)$ & 35.212 & 0.12 & 35.212 & $\mathrm{Ca}$ XI \\
\hline $35.566(16)$ & $0.15(7)$ & $35.562(12)$ & $0.26(7)$ & $35.566(16)$ & $0.23(14)$ & 35.576 & 0.27 & 35.576 & Ca XI \\
\hline $35.682(8)$ & $0.37(8)$ & $35.676(9)$ & $0.38(8)$ & $35.672(9)$ & $0.53(16)$ & 35.665 & 0.28 & 35.665 & S XIII \\
\hline $36.374(12)$ & $0.35(10)$ & $36.372(15)$ & $0.28(7)$ & $36.399(15)$ & $0.34(14)$ & 36.398 & 0.25 & 36.398 & S XII \\
\hline $36.544(19)$ & $0.15(6)$ & $36.561(19)$ & $0.29(9)$ & $36.547(15)$ & $0.24(13)$ & 36.563 & 0.24 & 36.563 & S XII \\
\hline
\end{tabular}

${ }^{a}$ Identifications for MEKAL (Mewe et al. 1995) and KELLY (Kelly 1987) identical.

${ }^{b}$ Observed flux in $10^{-4}$ photons $/ \mathrm{cm}^{2} / \mathrm{s}$.

${ }^{c}$ Model flux in $10^{-4}$ photons $/ \mathrm{cm}^{2} / \mathrm{s}$, obtained from 3-T fitting of LETGS (see Sect. 3.2).

${ }^{d}$ Fe XVII lines are strongly mixed with Fe XVI satellite lines.

${ }^{e}$ Line not split up; mixture of $15.175 \AA$ and $15.265 \AA$ from O VIII and Fe XVII, respectively.

${ }^{f}$ Small contamination by Fe XVIII possible.

Values in parentheses are statistical $1 \sigma$ uncertainties in the last digits.

and one pn). About half of the photons in the beams of two telescopes (Turner et al. 2001) are diffracted by sets of reflecting gratings and are then focussed onto the RGS detectors. The RGS spectral resolution is $\Delta \lambda \sim 0.07 \AA$, with a maximum effective area of about $140 \mathrm{~cm}^{2}$ around $15 \AA$. The wavelength uncertainty is $7-8 \mathrm{~m} \AA$. The first spectral order has been selected by means of the energy resolution of the individual CCDs. For further details see den Herder et al. (2001).
The data were processed by the XMM-Newton SAS using the calibration of February 2001. The RGS cover the range from 5 to $37 \AA$. The EPIC spectra, which have a lower resolution but higher sensitivity, are used to constrain the high-temperature part of Procyon's EM distribution. Because of the high resolution of the grating spectrometers we will focus on the spectra from these instruments. In Fig. 1, we show the RGS spectra together with an extract of the LETGS spectrum covering the 
wavelength range from 10 to $37 \AA$. No notable features are observed below $10 \AA$ in the LETGS and RGS spectra. However, the EPIC-MOS detects the $\mathrm{H}$ - and He-like lines of Mg. The remaining part of the LETGS spectrum is shown in Fig. 2. From Fig. 1 the gaps in the two RGS spectra due to CCD failure of CCD 7 (RGS1) and 4 (RGS2) are obvious.

\section{Analysis}

\subsection{Detailed analysis of the spectra}

The spectral lines from all three instruments have been measured individually. We folded monochromatic delta functions through the instrumental response matrices in order to derive the integrated line fluxes. No additional width was needed to fit the shape of the lines. A constant "background" level was adjusted in order to account for the real continuum and for the pseudo-continuum created by the overlap of several weak, neglected lines. In Table 1 , we have collected the measured wavelengths and fluxes of the emission lines in the RGS instruments together with those in the LETGS in the similar wavelength range. The fluxes among the three data sets, as collected in Table 1, are in good agreement in view of the systematic uncertainties in the calibration. However for some lines deviations appear, which are caused by gaps between the individual CCDs. In this wavelength range (below $40 \AA$ ) the identification is in general straightforward. The dominating lines are strong and belong to $\mathrm{H}-$ and He-like ions for which atomic parameters are well known. Although XMMNewton and Chandra observed Procyon at different dates no strong differences in flux (Table 1) are noticed, resulting in the conclusion that the coronal emission of Procyon did not vary strongly from one observation to the other.

Table 2 contains the same information as Table 1 for LETGS lines which occur above $37 \AA$. The extracted fluxes are as measured at Earth. Therefore they are not corrected for interstellar absorption which is of the order of $4 \%$ at $100 \AA, 6 \%$ at $125 \AA, 10 \%$ at $150 \AA$, and $15 \%$ at $175 \AA$. We added one Fe line (Fe X at $174.69 \AA)$ that was observed in an offset observation of Procyon (obsID $=1224$; $14.8 \mathrm{ksec})$. For that line the effective area was obtained by extrapolation. The line flux ratio of that line compared to the line at $171.075 \AA$ in the offset observation was used to establish the flux value.

In both tables, we have compared the measured wavelengths with the wavelengths in various atomic databases: the MEKAL (Mewe et al. 1985, 1995) $\operatorname{code}^{2}$, KELLY (Kelly 1987) and the database of the National Institute of Standards and Technology (NIST), which is also available on the web ${ }^{3}$. We have also compared with a list of lines observed in the solar corona (Doschek \& Cowan 1984, hereafter D\&C). Further we compare our measured iron lines with the results from laboratory experiments such as the Lawrence Livermore National Laboratory's Electron

\footnotetext{
${ }^{2}$ http://saturn.sron.nl/ kaastra/spex/line.ps.gz

3 http://physics.nist.gov/cgi-bin/AtData/main_asd
}

Beam Ion Trap (EBIT) (see Beiersdorfer et al. 1999 and Lepson et al. 2002 for Fe VIII-X and Lepson et al. 2000 for Fe XII-XIII). A number of lines in Table 2 (see note "d") are in close wavelength agreement to lines identified in EBIT. Finally in Table 1 the fluxes, from the multitemperature global fitting of Sect. 3.2, have been added.

Some possible line identifications have been omitted from Table 2, due to the absence of comparable lines belonging to the same multiplet or ion (Table 3) or due to ambiguity of the identification of lines in atomic databases (Kelly 1987). The latter concerns lines at $60.989 \AA$ (Si VII, VIII, \& IX) and $61.852 \AA$ (Si VIII \& IX).

Earlier benchmarks with a solar flare spectrum (Phillips et al. 1999) and with RGS and LETGS spectra of Capella (Audard et al. 2001a; Mewe et al. 2001) have already shown that the current atomic databases are lacking quite a number of spectral lines for L-shell transitions of $\mathrm{Ne}, \mathrm{Mg}, \mathrm{Si}$, and $\mathrm{S}$, that appear in the long-wavelength region above about $40 \AA$. This is illustrated by the many identifications present in the third column (KELLY), which are absent in MEKAL. For the Fe L-shell Behar et al. (2001) have shown that the HULLAC atomic data are fairly accurate.

\subsection{Global fitting and emission measure modeling}

\subsubsection{Multi-temperature fitting}

We first characterize the thermal structure and the elemental composition of Procyon's corona. To this end, we fitted multi-T models using SPEX (Kaastra et al. 1996a) of the spectra (RGS+MOS and LETGS). For both the observations the calculations require two dominant temperature components. A third (small and not very significant) temperature component is needed to account for the lines of low stages of ionization, present in the LETGS spectrum. The reduced $\chi^{2}$ is relatively high $(1.3-2)$ for the fits. This is due to a lack of lines in the MEKAL code and to the high resolution of the instrument. Small wavelength deviations (about 1-2 bins i.e. 0.02-0.04 $\AA$ ) between lines in the spectrum and in the model are often present (see Table 2). This effect results in a sharp maximum and minimum in the value of the normalized difference between model and observation around the peak of the line (see also Fig. 4). The results of RGS and LETGS are very similar.

In Table 4 results for temperatures $T$ (in MK), emission measures $E M$, and abundances are given. Statistic $1 \sigma$ uncertainties are given in parentheses. The emission measure is defined as $E M=n_{\mathrm{e}} n_{\mathrm{H}} V$, where $V$ is the volume contributing to the emission and for solar abundances the hydrogen density $n_{\mathrm{H}} \simeq 0.85 n_{\mathrm{e}}$. The temperatures and emission measures of all spectra show a dominant region between 1 and 2.5 MK. The two dominant temperature components are about 1.2 and 2.3 MK. Using EUVE, Schmitt et al. (1996) derived a DEM with a peak temperature of 1.6 MK based on Fe-lines only. This is in satisfactory agreement with our results. 
Table 2. Wavelengths and fluxes for LETGS above $36.5 \AA$, together with the line identifications from MEKAL, KELLY, and D\&C.

\begin{tabular}{|c|c|c|c|c|c|c|}
\hline \multicolumn{2}{|c|}{ LETGS } & \multicolumn{5}{|c|}{ 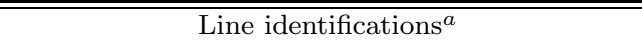 } \\
\hline & & \multicolumn{2}{|c|}{ MEKAL } & \multicolumn{2}{|c|}{ KELLY } & D\&C \\
\hline$\lambda(\AA)$ & flux $^{b}$ & $\lambda(\AA)$ & Ion & $\lambda(\AA)$ & Ion $\lambda(\AA)$ & Ion \\
\hline \multirow[t]{3}{*}{39.276} & $0.63(14)$ & 39.300 & S XI & 39.300 & S XI 39.30 & $\overline{\mathrm{S} \mathrm{XI}}$ \\
\hline & & & & 39.264 & Si X & \\
\hline & & & & 39.305 & Si X & \\
\hline 40.263 & $2.29(36)$ & 40.270 & $\mathrm{C} \mathrm{V}$ & 40.268 & C V 40.27 & $\mathrm{C} \mathrm{V}$ \\
\hline 40.718 & $1.88(42)$ & 40.730 & $\mathrm{C} \mathrm{V}$ & 40.731 & C V 40.73 & $\mathrm{C} \mathrm{V}$ \\
\hline \multirow[t]{2}{*}{41.475} & $1.07(29)$ & 41.470 & $\mathrm{C} \mathrm{V}$ & 41.472 & C V 41.47 & $\mathrm{C} \mathrm{V}$ \\
\hline & & 41.480 & Ar IX & 41.480 & Ar IX & \\
\hline 42.543 & $1.29(28)$ & 42.530 & S X & 42.543 & $\mathrm{~S} \times 42.53$ & S X \\
\hline 42.810 & $0.33(17)$ & - & & 42.826 & Si XI - & \\
\hline 43.743 & $0.54(8)$ & 43.740 & Si XI & 43.763 & Si XI 43.74 & Si XI \\
\hline 44.014 & $0.43(8)$ & 44.020 & Si XII & 44.021 & Si XII 44.02 & Si XII \\
\hline 44.150 & $0.67(10)$ & 44.165 & Si XII & & Si XII 44.17 & Si XII \\
\hline 44.218 & $0.52(10)$ & 44.249 & Si IX & 44. & Si IX 44.22 & Si IX \\
\hline \multirow[t]{2}{*}{45.677} & $0.20(4)$ & 45.680 & Si XII & 45.692 & Si XII 45.68 & Si XII \\
\hline & & & & 45.684 & Si X & \\
\hline 46.283 & $0.25(7)$ & 46.300 & Si XI & 46.300 & Si XI 46.30 & Si XI \\
\hline 46.391 & 0.4 & 46.410 & Si XI & & Si XI 4 & Si XI \\
\hline \multirow[t]{3}{*}{47.242} & $0.46(8)$ & 47.280 & $\operatorname{Mg} X$ & 47.310 & $\operatorname{Mg} X \quad 47.31$ & $\operatorname{Mg} X$ \\
\hline & & & & 47.231 & $\operatorname{Mg} X$ & \\
\hline & & & & 47.249 & S IX 47.25 & S IX \\
\hline \multirow[t]{3}{*}{47.452} & $0.48(8)$ & 47.500 & S IX & 47.433 & S IX 47.43 & S IX \\
\hline & & & & 47.518 & S IX & \\
\hline & & & & 47.453 & Si XI & \\
\hline \multirow[t]{2}{*}{47.642} & $0.49(8)$ & 47.654 & S X & 47.655 & S X 47.65 & S X \\
\hline & & & & 47.653 & Si XI & \\
\hline 47.774 & $0.34(7)$ & 47.793 & S X & 47.791 & S X 47.79 & S X \\
\hline \multirow[t]{2}{*}{47.883} & $0.24(8)$ & 47.896 & $\operatorname{Mg} X$ & 47.905 & S X 47.90 & $\operatorname{Mg} X$ \\
\hline & & & & 47. & Si XI & \\
\hline 48.720 & $0.23(6)$ & 48.730 & Ar IX & 48.73 & Ar IX 48.73 & Ar IX \\
\hline 49.109 & 0.3 & - & & 49 & S IX 49.12 & S IX \\
\hline \multirow[t]{2}{*}{49.207} & $1.44(14)$ & 49.220 & Si XI & 49.222 & Si XI 49.22 & Si XI \\
\hline & & 49.180 & Ar IX & 49.18 & Ar IX 49.18 & Ar IX \\
\hline 49.324 & $0.32(7)$ & - & & 49.328 & S IX - & \\
\hline 49.696 & $0.29(7)$ & - & & 49.701 & Si X - & \\
\hline 49.975 & $0.28(6)$ & 50.019 & Si VIII ${ }^{c}$ & 50.019 & Si VIII - & \\
\hline 50.327 & $0.51(8)$ & - & & 50.333 & Si X - & \\
\hline 50.520 & $1.68(15)$ & 50.530 & Si X & 50.524 & Si X 50.53 & Si X \\
\hline 50.686 & $1.30(14)$ & 50.690 & Si X & 50.691 & Si X 50.69 & Si X \\
\hline 52.306 & $0.75(11)$ & 52.300 & Si XI & 52.296 & Si XI 52.30 & Si XI \\
\hline 52.594 & $=0.35(8)$ & 52.615 & Ni XVIII & 52.615 & Ni XVIII - & \\
\hline & & & & 52.611 & $\mathrm{Si} I X$ & \\
\hline 52.715 & $0.30(7)$ & 52.720 & Ni XVIII & 52.720 & Ni XVIII 52.70 & S VIII \\
\hline \multirow[t]{2}{*}{52.772} & $0.30(7)$ & - & & 52.756 & S VIII - & \\
\hline & & & & 52.789 & S VIII & \\
\hline 52.898 & $0.30(7)$ & 52.911 & Fe XV & 52.911 & Fe XV 52.87 & Fe XV \\
\hline \multirow[t]{2}{*}{54.133} & $0.56(13)$ & 54.118 & S VIII & 54.118 & S VIII 54.12 & S VIII \\
\hline & & 54.142 & Fe XVI & 54.142 & Fe XVI 54.15 & Fe XVI \\
\hline 54.180 & $0.31(10)$ & 54.180 & S IX & 54.175 & S IX 54.18 & S IX \\
\hline \multirow[t]{2}{*}{54.546} & $0.54(13)$ & - & & 54.571 & Si X - & \\
\hline & & & & 54.566 & S VIII & \\
\hline 54.700 & 0.45 & 54. & Fe XVI & 54.728 & Fe XVI 54.70 & Fe XVI \\
\hline 55.094 & $0.68(15)$ & 55.060 & Mg IX & 55.060 & Mg IX 55.06 & Mg IX \\
\hline & & & & 55.094 & Si IX 55.12 & Si IX \\
\hline & & & & 55.116 & Si IX & \\
\hline & & & & 55.096 & Si X & \\
\hline 55.270 & $0.88(25)$ & 55.272 & Si IX & 55.272 & Si IX 55.27 & Si IX \\
\hline & & & & 55.305 & Si IX 55.31 & Si IX \\
\hline
\end{tabular}

Table 2. continued.

\begin{tabular}{llrrrr}
\hline \hline \multicolumn{2}{c}{ LETGS } & \multicolumn{4}{c}{ Line identifications $^{a}$} \\
\hline \multicolumn{5}{c}{ MEKAL } & \multicolumn{2}{c}{ KELLY $^{c}$ D\&C } \\
\hline$\lambda(\AA)$ & flux $^{b}$ & $\lambda(\AA)$ & Ion $\lambda(\AA)$ & Ion $\lambda(\AA)$ & Ion \\
\hline 55.359 & $2.14(27)$ & 55.356 & Si IX 55.356 & Si IX 55.36 & Si IX \\
& & & 55.401 & Si IX 55.40 & Si IX \\
56.037 & $0.19(11)$ & 56.000 & Ni XIII 56.027 & Si IX 56.03 & Si IX \\
& & & 56.081 & S IX 56.08 & S IX \\
56.836 & $0.20(8)$ & - & 56.804 & Si X - & \\
57.741 & $0.80(35)-$ & 57.736 Mg VIII - &
\end{tabular}

$57.7410 .80(35)-$

$\begin{array}{llllll}57.856 & 0.78(35) & 57.880 & \text { Mg X } 57.876 & \text { Mg X } 57.88 & \text { Mg X }\end{array}$ $\begin{array}{llll}57.920 \quad M g X & 57.920 \quad M g X & 57.92 & M g X\end{array}$ $61.0201 .41(25) 61.050 \quad$ Si VIII 61.019 Si VIII $61.01 \quad$ Si VIII $61.0871 .38(24)-$ $61.038 \mathrm{Mg}$ IX 61.070 Si VIII $61.08 \quad$ Si VIII $61.088 \mathrm{Mg}$ IX

$\begin{array}{llllll}61.578 & 0.52(17) & 61.600 & \text { S VIII } 61.600 & \text { S VIII } 61.60 & \text { S VIII }\end{array}$ $\begin{array}{llll}61.668 & 0.49(15)- & 61.649 & \text { Si IX } 61.66\end{array}$ Si IX $\begin{array}{lllll}61.843 & 0.67(11) & 61.841 & \text { Si IX } 61.852 & \text { Si IX } 61.84\end{array}$ Si IX

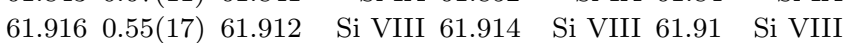
61.895 Si VIII $61.90 \quad \mathrm{Si}$ VIII $\begin{array}{llllll}62.748 & 0.53(17) & 62.755 & \mathrm{Mg} \text { IX } & 62.751 & \mathrm{Mg} \text { IX }\end{array} 62.76 \quad \mathrm{Mg}$ IX $62.699 \mathrm{Fe} \mathrm{XIII}^{d}$ 62.694 Fe XIII

$\begin{array}{lllll}62.849 & 0.38(11) & 62.879 & \text { Fe XVI } 62.879 & \text { Fe XVI } 62.88 \text { Fe XVI }\end{array}$ $62.800 \quad \mathrm{Fe} \mathrm{X}^{d} 62.8 \quad$ Fe X

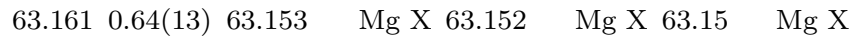

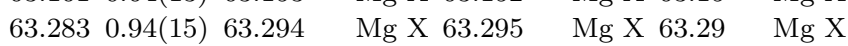

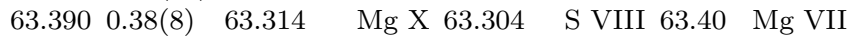
$63.396 \mathrm{Mg}$ VII

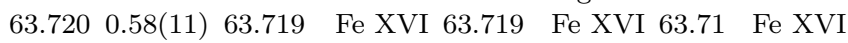
63.732 Si VIII $63.73 \quad$ Si VIII

$63.9210 .39(10)-$

$64.1350 .44(11)-$

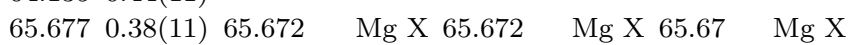

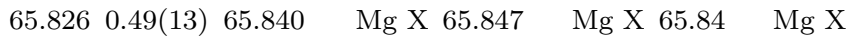
65.822 Ne VIII

$65.8840 .41(10) 65.905 \quad$ Fe XII $^{d} 65.905 \quad$ Fe XII 65.892 Ne VIII -

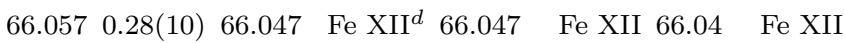
$66.2550 .64(14)-$

$66.3520 .63(13)-$

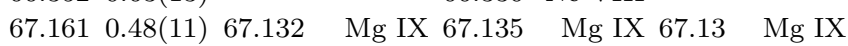

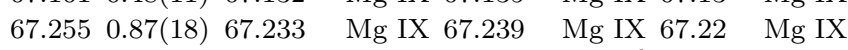
$67.291 \mathrm{Fe} \mathrm{XII}^{d}$

$67.3750 .68(14) 67.350$ Ne VIII 67.382 Ne VIII 67.35 Ne VIII

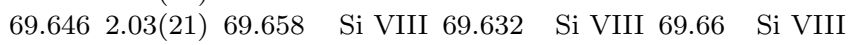
69.660 Fe XV 69.66 Fe XV

69.827 1.05(14) $69.825 \quad$ Si VIII $69.790 \quad$ Si VIII $69.83 \quad$ Si VIII

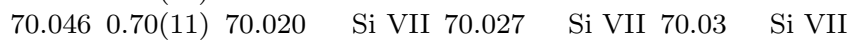
70.010 Fe XII 70.01 Fe XII 70.054 Fe XV 70.054 Fe XV 70.05 Fe XV

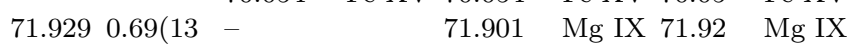
71.955 Si VII

$\begin{array}{llllll}72.034 & 0.43(13) & 72.030 & \text { Mg IX } 72.027 & \text { Mg IX } 72.03 & \text { Mg IX }\end{array}$

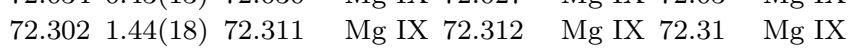
72.310 Fe XI $72.310 \quad$ Fe XI

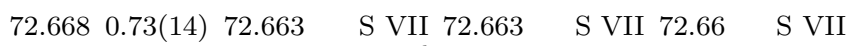
$\begin{array}{lllll}72.871 & 0.58(15) & 72.850 & \text { Fe IX }^{d} 72.850 \quad \text { Fe IX - }\end{array}$

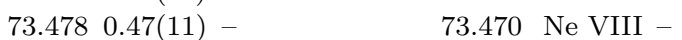
73.471 Fe XV 73.471 Fe XV 73.47 Fe XV $73.5550 .43(11) 73.560$ Ne VIII 73.563 Ne VIII 73.56 Ne VIII $74.8601 .10(18) 74.854 \mathrm{Mg}$ VIII $74.858 \mathrm{Mg}$ VIII $74.85 \mathrm{Mg}$ VIII 74.845 Fe XIII $^{d} 74.845$ Fe XIII 
Table 2. continued.

\begin{tabular}{|c|c|c|c|c|c|c|c|}
\hline \multirow{2}{*}{\multicolumn{2}{|c|}{ LETGS }} & \multicolumn{6}{|c|}{ Line identifications $^{a}$} \\
\hline & & \multicolumn{2}{|c|}{ MEKAL } & \multicolumn{2}{|c|}{ KELLY } & \multicolumn{2}{|r|}{$\mathrm{D \& C}$} \\
\hline$\lambda(\AA)$ & flux $^{b}$ & $\lambda(\AA)$ & Ion & $\lambda(\AA)$ & Ion & $\lambda(\AA)$ & Ion \\
\hline 5.035 & $1.05(18)$ & 75.034 & Mg VIII & 75.034 & Ig VIII & 75.03 & Mg VIII \\
\hline .978 & $0.47(11)$ & 76.006 & $\mathrm{Fe} \mathrm{X}^{d}$ & 76.006 & $\mathrm{Fe} X$ & 76.02 & $\mathrm{Fe} \mathrm{X}$ \\
\hline .038 & $0.77(13)$ & - & & - & & - & \\
\hline .507 & $0.32(11)$ & 76.502 & Fe XVI & 76.502 & Fe XVI & 76.51 & Fe XVI \\
\hline .862 & $0.55(13)$ & - & & - & & 76.87 & Fe XVI \\
\hline 7.740 & $1.11(18)$ & 77.741 & Mg IX & 77.737 & Mg IX & 77.74 & Mg IX \\
\hline \multirow[t]{2}{*}{78.733} & $0.71(14)$ & 78.717 & $\mathrm{Ni} \mathrm{XI}$ & 78.744 & Ni XI & 78.72 & Ni XI \\
\hline & & 78.769 & $\mathrm{Fe} \mathrm{X}^{d}$ & 78.769 & Fe X & & \\
\hline .483 & $0.58(13)$ & 79.488 & Fe XII & 88 & Fe XII & 79.49 & Fe XII \\
\hline .017 & 0.38( & 80.022 & $\mathrm{Fe}$ XII & 80.022 & X XII & 80.02 & Fe XII \\
\hline 80.236 & $0.54(14)$ & - & & 80.255 & Mg VIII & - & \\
\hline \multirow[t]{2}{*}{80.507} & $0.74(14)$ & 80.501 & Si VI & 80.501 & Si VI & 80.50 & Si VI \\
\hline & & 80.510 & Fe XII & & Fe XII & 80.51 & Fe XII \\
\hline 80.751 & $0.54(14)$ & - & & 80.725 & Si VI & - & \\
\hline .865 & 0.42( & - & & 81.895 & Si VII & - & \\
\hline 82.420 & $0.48(11)$ & 82.430 & $\mathrm{Fe} \mathrm{IX}^{d}$ & 82.430 & Fe IX & 82.43 & Fe IX \\
\hline 82.667 & 0.97( & 82.744 & Fe XII & 82.598 & Mg VIII & - & \\
\hline \multirow[t]{2}{*}{82.808} & $0.35(10)$ & 82.837 & Fe XII & 82.837 & Fe XII & - & \\
\hline & & & & 82.822 & Mg VIII & & \\
\hline 83.337 & $0.38(11)$ & - & & 83.358 & Si VI & - & \\
\hline \multirow[t]{2}{*}{83.600} & 0.55 & - & & 83.587 & Mg VII & 83.59 & $\mathrm{Mg}$ VII \\
\hline & & & & 83.611 & Si VI & & \\
\hline 83.764 & 0.47 & 83.766 & Mg VII & & Mg VII & 83.77 & Mg VII \\
\hline \multirow[t]{2}{*}{83.935} & 0.46 & 83.959 & Mg VII & & VII & 83.96 & $\mathrm{Mg}$ VII \\
\hline & & & & & VII & & Mg VII \\
\hline 84.032 & 0.40 & - & & 84 & VII & 84.02 & Mg VII \\
\hline \multirow[t]{2}{*}{84.292} & 0.40 & 84.292 & Ne VII & 84.292 & $\mathrm{Ne}$ VII & 84.29 & $\mathrm{Ne}$ VII \\
\hline & & & & 84.212 & $\mathrm{Ne}$ VII & & \\
\hline 84.433 & 0.3 & - & & - & & - & \\
\hline \multirow[t]{2}{*}{85.448} & 0.38 & - & & 85 & II & 85.47 & Fe XII \\
\hline & & & & & VII & 41 & Mg VII \\
\hline 765 & 1.1 & 86. & Fe XI & & $\mathrm{Fe} \mathrm{XI}$ & 86.77 & $\mathrm{Fe} \mathrm{XI}$ \\
\hline 86.876 & 0.55 & - & & & Mg VIII & - & \\
\hline \multirow[t]{2}{*}{87.021} & 0.46( & 87.025 & $\mathrm{Fe} \mathrm{XI}$ & & $\mathrm{Fe} \mathrm{XI}$ & 87.02 & $\mathrm{Fe} \mathrm{XI}$ \\
\hline & & & & & g VIII & & \\
\hline 7 & & 92 & $\mathrm{Ne}$ VIII & & Ne VIII & 88.08 & $\mathrm{Ne}$ VIII \\
\hline & $0.6 \varepsilon$ & - & & - & & - & \\
\hline 955 & 0.75 & - & & & g VI & - & \\
\hline & 0.43 & 89.185 & $\mathrm{Fe} \mathrm{XI}$ & & Fe XI & 89 . & $\mathrm{Fe} \mathrm{XI}$ \\
\hline 9 & 0.5 & - & & & Mg VII & - & \\
\hline \multirow[t]{2}{*}{90.989} & $0.43(10)$ & 91.009 & Fe XIV & & XIV & - & \\
\hline & & & & & & & \\
\hline & & 91 & $\mathrm{Ne}$ VII & 91 & VII & 91.56 & $\mathrm{Ne}$ VII \\
\hline & & - & & - & & - & \\
\hline & 0.5 & 91. & $\mathrm{NiX}$ & & $\mathrm{X}$ & 91. & $\mathrm{Ni} Y$ \\
\hline & & - & & & III & - & \\
\hline & 0.5 & - & & 92. & $\mathrm{Ne}$ VII & - & \\
\hline & 0.5 & - & & - & & - & \\
\hline & & & $\mathrm{Fe} \mathrm{X}^{d}$ & & $\mathrm{X}$ & & Fe X \\
\hline 39 & $0.90(18)$ & 374 & $\mathrm{Fe} \mathrm{X}$ & & $\mathrm{Fe} \mathrm{X}$ & 95. & e $Y$ \\
\hline & & & $\mathrm{Fe} \mathrm{X}^{d}$ & & $\mathrm{X}$ & - & \\
\hline 95.412 & $1.04(20)$ & 95.483 & Mg VI & 83 & VI & 95 & $\mathrm{Mg}$ VI \\
\hline & & & & & & & Mg VI \\
\hline & & - & & & Si VI & 96.02 & $\mathrm{Si}$ VI \\
\hline & & & $\mathrm{Fe} \mathrm{X}^{d}$ & & Fe X & 96. & $\mathrm{Fe} X$ \\
\hline & & & $\mathrm{Fe} \mathrm{X}^{d}$ & 96 & Fe X & - & \\
\hline & & & $\mathrm{Fe} \mathrm{X}^{d}$ & & Fe X & & e X \\
\hline & & & $\mathrm{Ne}$ VII & & & & e VII \\
\hline & & & & & & & $\mathrm{Ne}$ VIII \\
\hline 98.251 & $2.89(34)$ & 98.260 & Ne VIII & 260 & VIII & 98.26 & Ne VIII \\
\hline
\end{tabular}

Table 2. continued.

\begin{tabular}{|c|c|c|c|c|c|c|c|}
\hline \multirow{2}{*}{\multicolumn{2}{|c|}{ LETGS }} & \multicolumn{6}{|c|}{ Line identifications $^{a}$} \\
\hline & & \multicolumn{2}{|c|}{ MEKAL } & \multicolumn{2}{|c|}{ KELLY } & \multicolumn{2}{|c|}{$\mathrm{D \& C}$} \\
\hline$\lambda(\AA)$ & flux $^{b}$ & $\lambda(\AA)$ & Ion & $\lambda(\AA)$ & Ion & $\lambda(\AA)$ & Ion \\
\hline 100.57 & $1.05(24)$ & - & & 100.597 & Mg VIII & - & \\
\hline .85 & $0.90(22)$ & 102.91 & $\mathrm{Ne}$ VIII & 911 & $\mathrm{Ne}$ VIII & 102.9 & $\mathrm{Ne}$ VIII \\
\hline .07 & 1.68 & 3.08 & $\mathrm{Ne}$ VIII & 085 & $\mathrm{Ne}$ VIII & 103.1 & $\mathrm{Ne}$ VIII \\
\hline 103.54 & 2.08 & 103.57 & $\mathrm{Fe} \mathrm{IX}^{d}$ & & e IX & 103.6 & $\mathrm{Fe}$ IX \\
\hline 3.88 & $0.72(22)$ & - & & - & & - & \\
\hline 4.67 & 0.68 & - & & - & & - & \\
\hline 4.78 & $0.86(21)$ & .81 & O VI & & O VI & & \\
\hline 105.20 & 1.22 & 21 & $\mathrm{Fe} \mathrm{IX}^{d}$ & & Fe IX & 105.2 & Fe IX \\
\hline 106.18 & 1.03( & 106.19 & Ne VII & & Ne VII & 106.2 & $\mathrm{Ne}$ VIl \\
\hline 111.23 & $1.49(28)$ & - & & & $\mathrm{Ca} \mathrm{X}$ & - & \\
\hline 111.71 & $0.53(13)$ & 111.57 & Mg VI & & $\mathrm{Mg}$ VI & 111.6 & $\mathrm{Mg} \mathrm{Vl}$ \\
\hline & & 111.72 & Mg VI & & VI & 111.7 & $\mathrm{Mg} \mathrm{Vl}$ \\
\hline 113.33 & $0.46(11)$ & - & & 315 & Fe VIII & - & \\
\hline 113.77 & $0.60(20)$ & - & & 113.763 & Fe VIII & & \\
\hline & & & & 793 & Fe IX & & \\
\hline 113.99 & $0.71(20)$ & - & & 113.990 & $\mathrm{Mg} \mathrm{V}$ & 114.0 & $\mathrm{Mg} \mathrm{V}$ \\
\hline & & & & 114.029 & $\mathrm{Mg} \mathrm{V}$ & & \\
\hline 114.54 & $0.48(14)$ & - & & 114.564 & Fe VIII & - & \\
\hline 114.88 & $0.66(17)$ & - & & 114.785 & $\mathrm{Mg} \mathrm{V}$ & 114.8 & $\mathrm{Mg} \mathrm{V}$ \\
\hline 115.37 & $0.51(18)$ & 115.33 & $\mathrm{Ne}$ VII & 115.33 & $\mathrm{Ne}$ VII & - & \\
\hline & & - & & 115.39 & $\mathrm{Ne}$ VII & - & \\
\hline 115.80 & $0.77(20)$ & 115.83 & $\mathrm{O} \mathrm{VI}$ & 115.826 & O VI & 115.8 & $\mathrm{O} \mathrm{Vl}$ \\
\hline
\end{tabular}

$\begin{array}{lll}115.89 & 0.46(17)-\end{array}$

$\begin{array}{lllll}116.70 & 1.54(25) & 116.69 & \text { Ne VII } 116.693 & \text { Ne VII } 116.7 \quad \text { Ne VII }\end{array}$ $116.870 .54(14)-$

$117.200 .54(20) \quad 117.20 \mathrm{Fe} \mathrm{VIII}^{d} 117.197$ Fe VIII -

$117.660 .80(20)-$

$119.310 .46(13)-$

$120.310 .60(15) 120.33 \quad$ O VII $120.331 \quad$ O VII -

$\begin{array}{lllll}122.49 & 0.73(18) & 122.49 & \text { Ne VI } 122.49 & \text { Ne VI } 122.5\end{array}$

$123.541 .31(31)-$

$124.510 .98(25)-$

$\begin{array}{lll}126.25 & 0.98(32)-\end{array}$

$127.530 .98(25)-$

$\begin{array}{lllll}127.69 & 1.44(29) & 127.66 & \text { Ne VII } 127.663 & \text { Ne VII } 127.7\end{array}$ Ne VII

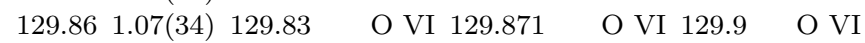
$130.921 .78(39) 130.94 \mathrm{Fe} \mathrm{VIII}^{d} 130.941$ Fe VIII 130.9 Fe VIII

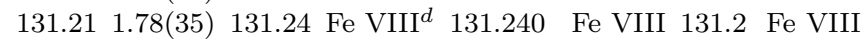
$134.211 .43(41)-$

$\begin{array}{lllllll}135.48 & 0.97(35) & 135.52 & \text { O V } 135.523 & \text { O V } 135.5 & \text { O V }\end{array}$ $136.781 .91(53)-$ $140.271 .36(49)-$ $\begin{array}{lll}141.04 & 0.79(22) & 141.04\end{array}$ $144.97 \quad 1.73(49) 144.99$ $\begin{array}{lll}147.27 & 1.12(35) & 147.27\end{array}$ $\begin{array}{lll}148.36 & 11.3(10) & 148.40\end{array}$ $150.085 .08(60) 150.10$ $151.522 .36(39)-$

$\begin{array}{lll}152.11 & 5.60(66) & 152.15\end{array}$

Ca XII 141.038

$\mathrm{Ni} X 144.988$

Ca XII 147.278

Ni XI 148.402

O VI 150.1

151.548

$154.142 .73(42) 154.18$

$155.561 .36(28)-$

$156.141 .66(32)-$

$156.381 .29(31)-$

$\begin{array}{lllllll}157.68 & 2.76(42) & 157.73 & \text { Ni XIII } & 157.730 & \text { Ni XIII } 157.7 & \text { Ni XIII }\end{array}$

$\begin{array}{lllll}158.33 & 2.08(35) & 158.38 & \text { Ni X } 158.377 & \text { Ni X } 158.4\end{array}$ $158.781 .47(34)-$ $159.241 .03(27)-$ $\begin{array}{lll}159.58 & 1.83(43)-\end{array}$

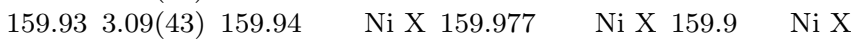
159.97 Ni XIII $159.97 \quad$ Ni XIII

Ca XII 141.0 Ca XII Ni X $145.0 \quad$ Ni X Ca XII 147.3 Ca XII Ni XI 148.4 Ni XI O VI $150.1 \quad$ O VI $\mathrm{O} \mathrm{V}-$

Ni XII 152.153 Ni XII 152.2 Ni XII Ni XII 154.175 Ni XII 154.2 Ni XII 156.140 Ne V 158.770 Ni XIII -

159.300 Si X 159.1 Ar XIII

\begin{tabular}{rrr}
159.97 & Ni XIII $159.97 \quad$ Ni XIII \\
\hline
\end{tabular}


Table 2. continued.

\begin{tabular}{|c|c|c|c|}
\hline \multirow[t]{2}{*}{ LETGS } & \multicolumn{3}{|c|}{ Line identifications $^{a}$} \\
\hline & MEKAL & & $\& \mathrm{C}$ \\
\hline$\lambda(\AA) \quad$ flux $^{b}$ & Ion $\lambda(\AA)$ & Ion $\lambda(\AA)$ & Ion \\
\hline $162.562 .94(83)$ & N V 162.556 & $\mathrm{~N} \mathrm{~V}-_{-}$ & \\
\hline $164.113 .53(74)$ & 164.15 Ni XIII 164.146 & Vi XIII 16 & XIII \\
\hline $167.433 .9(10)$ & 167.49 Fe VIII 167.486 & e VIII 167. & e VIII \\
\hline $167.593 .9(12)$ & 167.66 Fe VIII 167.656 & e VIII - & \\
\hline $168.137 .4(13)$ & 168.17 Fe VIII 168.172 & e VIII 168.2 & Fe VIII \\
\hline $168.515 .4(13)$ & 168.54 Fe VIII 168.545 & e VIII 168.5 & Fe VIII \\
\hline $168.905 .0(18)$ & $168.93 \mathrm{Fe}$ VIII 168.929 & e VIII 168.9 & Fe VIII \\
\hline $171.04114(8)$ & $171.08 \quad$ Fe IX 171.075 & Fe IX 171.1 & $\mathrm{Fe} \mathrm{IX}$ \\
\hline $174.49118(24)$ & Fe X 174.53 & Fe X 174.5 & $\mathrm{Fe} X$ \\
\hline
\end{tabular}

${ }^{a}$ From MEKAL (Mewe et al. 1995), KELLY (1987), and D\&C (solar line list of Doschek \& Cowan 1984).

${ }^{b}$ Observed flux in $10^{-4}$ photons $/ \mathrm{cm}^{2} / \mathrm{s}$ with in parentheses $1 \sigma$ uncertainty in the last digits.

${ }^{c}$ MEKAL placed it at $52.0 \AA$.

${ }^{d}$ Line identified in EBIT spectrum.

${ }^{e}$ From CHIANTI (Dere et al. 1997).

The total emission measures summed over all temperature components are about $4.6(.4) \times 10^{50} \mathrm{~cm}^{-3}$ for LETGS and $3.9(.3) \times 10^{50} \mathrm{~cm}^{-3}$ for RGS+MOS. These are similar to the total $E M$ of $4.5 \times 10^{50} \mathrm{~cm}^{-3}$ found by Schmitt et al. (1996).

The determination of abundances is complicated by several factors. The many weak L-shell lines, which are absent in the atomic code (see difference between Col. "MEKAL" and "KELLY" of Table 2) can produce a "pseudo-continuum" (see e.g. Fig. 2a between 42 and $58 \AA$ ), which bias the determination of the real but very weak continuum. Several fits to the LETGS spectrum were made: a) to the total spectrum, b) to the total spectrum with selected lines in the wavelength range from 40 to $100 \AA$, to limit the influence of the inaccuracy of atomic data of Ne-, Mg-, and Si- L-shell lines, and c) to a line spectrum with lines of Table 1 and lines with a statistical significance $\gtrsim 4 \sigma$ in the wavelength range above $40 \AA$ (see Table 5). During our investigations the absolute (relative to $\mathrm{H}$ ) abundances turned out to be very sensitive to the selected group of elements introduced in the fit procedure. This is especially true for the elements Ar and Ca. For these reasons no consistent absolute values of the abundances could be obtained. However, abundance ratios turn out to be much more robust. Therefore the abundance values are normalized to oxygen, and are given relative to their solar photospheric values (Anders \& Grevesse 1989), except for iron. For Fe we use $\log A_{\mathrm{Fe}}$ is 7.51 (see Drake et al. 1995) instead of 7.67 (Anders \& Grevesse 1989). Here $\log A_{\mathrm{Fe}}$ is the logarithmic of the Fe-abundance relative to $\log A_{\mathrm{H}}=12.0$. The abundances presented in Table 4 are derived assuming the same abundances for the three temperature components. These are averaged over the different fits, together with their least-squares-fit standard deviations (within parentheses).
Table 3. Possible line identifications left out of Table 2. Col. $\lambda$ : observed wavelengths from Table 2. Columns 2 and 3 give a possible identification which has not been given in Table 2, due to the absence of the lines, given in Cols. 4 and 5.

\begin{tabular}{lrlrl}
\hline \hline$\lambda(\AA)$ & present & ion & missing & ion \\
\hline 93.587 & 93.616 & FeVIII & 93.469 & FeVIII \\
& & & 108.077 & FeVIII \\
98.583 & 98.548 & FeVIII & 98.371 & FeVIII \\
103.88 & 103.937 & FeXVIII & 93.923 & FeXVIII \\
103.88 & 103.904 & MgV & 110.859 & MgV \\
\hline
\end{tabular}

We obtain abundance values between 0.7 and 2.4 relative to oxygen (e.g., some enhancement for $\mathrm{Ne}$ and $\mathrm{Si}$ ). However, apart from statistical errors these values are also sensitive to systematic errors, due to changes in values of the solar photospheric abundances, where uncertainties of a factor of 2 cannot be excluded (e.g., Prieto et al. 2001; Grevesse \& Sauval 1998). So we cannot obtain indications for a significant FIP effect (enhancement of elements with a low First Ionization Potential) as found for the solar corona (e.g., Feldman et al. 1992). This confirms the conclusions by Drake et al. (1995), based on relative abundances from EUVE observations. The abundances of $\mathrm{C}$ and $\mathrm{N}$, relative to $\mathrm{O}$ are somewhat higher than the values obtained in the solar photosphere (Anders \& Grevesse 1989). In the EUVE observations by Drake et al. (1995) no suitable C- and N-lines were present to constrain (relative) abundances.

Values for $n_{\mathrm{e}}$, given in Table 4 , have been obtained by fitting to the O VII and N VI triplet lines. The C V lines have been omitted from this procedure because their intensities are sensitive for the stellar UV-radiative field, mimicing higher densities (Ness et al. 2001; Porquet et al. 2001).

\subsubsection{Temperature dependent emission measure modeling}

To show the connectivity of the different temperature components we applied a differential emission measure (DEM) model of Procyon's corona using the various inversion techniques offered by SPEX (see Kaastra et al. 1996b). We applied the abundances obtained in Sect. 3.2.1. In Fig. 3 we give the results based on the regularisation method. Other inversion methods (smoothed clean, or polynomial) give statistically comparable results. The DEM modeling has been applied separately to RGS+MOS and to LETGS.

As a result we find a dominant emission measure of the order of $10^{50} \mathrm{~cm}^{-3}$ between $1-3 \mathrm{MK}$. The total emission measures are $3.5(.3) \times 10^{50} \mathrm{~cm}^{-3}$ for RGS+MOS and $4.5(.2) \times 10^{50} \mathrm{~cm}^{-3}$ for LETGS (in line with the multitemperature fitting). Figure 3 allows us to conclude that there is no significant amount of $E M$ at $T \gtrsim 4 \mathrm{MK}$ in the corona of Procyon. Schmitt et al. (1996) give an upper limit of $6 \mathrm{MK}$, based on EUVE observations. The $E M$ 
Table 4. Best-fit parameters for a 3-T CIE model fit. Elemental abundances for the three instruments are given normalized to oxygen and relative to solar photospheric values given by Anders \& Grevesse (1989), except for $\mathrm{Fe}^{a}$. $1 \sigma$ uncertainties are given in brackets.

\begin{tabular}{lll}
\hline \hline Parameter & LETGS & RGS+MOS \\
\hline $\log N_{\mathrm{H}}\left[\mathrm{cm}^{-2}\right]$ & $18.06^{b}$ & $18.06^{b}$ \\
$T_{1}[\mathrm{MK}]$ & $0.63(.10)$ & - \\
$T_{2}[\mathrm{MK}]$ & $1.21(.07)$ & $1.65(.15)$ \\
$T_{3}[\mathrm{MK}]$ & $2.26(.12)$ & $2.68(.22)$ \\
$E M_{1}\left[10^{50} \mathrm{~cm}^{-3}\right]$ & $0.41(.14)$ & - \\
$E M_{2}\left[10^{50} \mathrm{~cm}^{-3}\right]$ & $2.45(.27)$ & $3.0(.20)$ \\
$E M_{3}\left[10^{50} \mathrm{~cm}^{-3}\right]$ & $1.72(.29)$ & $0.9(.18)$ \\
$n_{\mathrm{e} 2}\left[10^{10} \mathrm{~cm}^{-3}\right]$ & $1.4+1.5$ & $1.5_{-0.6}^{+2.0}$ \\
$n_{\mathrm{e} 3}\left[10^{10} \mathrm{~cm}^{-3}\right]$ & $0.2{ }_{-0.2}^{+0.8}$ & - \\
$\mathrm{O} / \mathrm{H}$ & $0.68(0.38)$ & $0.76(0.33)$ \\
$\mathrm{C} / \mathrm{O}$ & $1.38(.24)$ & $1.45(.29)$ \\
$\mathrm{N} / \mathrm{O}$ & $1.33(.10)$ & $1.47(.5)$ \\
$\mathrm{O} / \mathrm{O}$ & 1.0 & 1.0 \\
$\mathrm{Ne} / \mathrm{O}$ & $1.49(.21)$ & $1.53(.27)$ \\
$\mathrm{Mg} / \mathrm{O}$ & $1.1(.5)$ & - \\
$\mathrm{Si} / \mathrm{O}$ & $1.56(.36)$ & - \\
$\mathrm{S} / \mathrm{O}$ & $0.69(.15)$ & - \\
$\mathrm{Fe} / \mathrm{O}$ & $0.97(.31)$ & $1.47(.22)$ \\
$\mathrm{Ni} / \mathrm{O}$ & $2.39(.27)$ & - \\
\hline
\end{tabular}

${ }^{a}$ In logarithmic units, with $\log _{10} \mathrm{H}=12.00 ; \mathrm{C}=8.56 ; \mathrm{N}=$ $8.05 ; \mathrm{O}=8.93 ; \mathrm{Ne}=8.09 ; \mathrm{Mg}=7.58 ; \mathrm{Si}=7.55 ; \mathrm{S}=7.21$; $\mathrm{Ar}=6.56 ; \mathrm{Ca}=6.36 ; \mathrm{Fe}=7.51$ (see text) $\mathrm{Ni}=6.25$.

${ }^{b}$ See Linsky et al. (1995).

observed at different times as well as lines fluxes in Table 1 show no significant variability.

Figure 4 shows fit residuals of parts of the LETGS spectrum fitted using this temperature-dependent emission measure modeling, i.e. applying the model of Fig. 3 (LETGS). From Fig. 4a we recognize large deviations in residual due to model insufficiencies and a pseudocontinuum (most fit residuals positive) due to the lack of weaker lines in current atomic databases in this wavelength range. Clear from Fig. 4b are the succeeding large positive and negative residuals around 148 and $171 \AA$, due to wavelength deviations of lines in the spectrum and the model.

\subsection{Consistency checks using individual lines}

The question is whether the model insufficiencies influence our conclusions about temperatures, emission measures, and abundances as obtained in Sect. 3.2. Therefore we have also compared observed and model line fluxes. The advantage of this individual line approach is that we can select strong and unblended lines, for which the theoretical emissivities are quite well established.

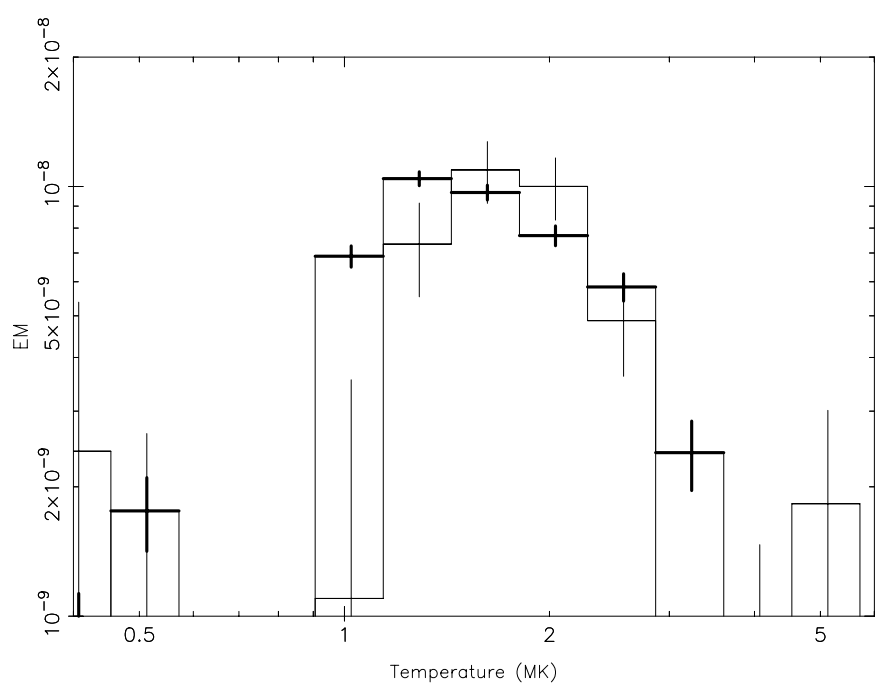

Fig. 3. EM $\left(n_{\mathrm{e}} n_{\mathrm{H}} V\right.$ in $\left.10^{64} \mathrm{~m}^{-3}\right)$ for RGS and LETGS (thick), using the regularisation algorithm. The relative abundances given in Table 4 have been applied.

For the short-wavelength region this is done for all lines (Table 1), while for the longer wavelength range only lines with a statistical significance $\gtrsim 4 \sigma$ were used. For the latter the fluxes have been compared with the 3 -T model as well as with the results from the DEM model. The values are given in Table 5. This table shows generally a good agreement between the observed flux and the 3-T flux and the flux from the DEM-modeling, summed over the T-bins. Most striking are the deviations for the Fe VIII lines around 131 and $168 \AA$. This is definitely due to a large deficiency in the atomic data used. From atomic physics grounds the line at $168.13 \AA$ is the stronger, as observed in the spectrum and in laboratory experiments (Wang et al. 1984), but in our code this line turns out to be the weak$\mathrm{est}^{4}$. Another interesting feature is the contamination of the forbidden $\mathrm{C} \mathrm{V}$ line - which is often used for density diagnostics - with Ar IX. Another clear example of blending is the line at $74.860 \AA$ which contains Mg VIII and Fe XIII.

We have measured line ratios of density-sensitive Helike triplets from the LETGS and RGS spectra, taking into account the photo-exciting UV flux (Porquet et al. 2001). Our results are consistent in both instruments $\left(n_{\mathrm{e}} \approx 10^{10} \mathrm{~cm}^{-3}\right)$ and similar to those of Ness et al. (2001) and our values given in Table 4 . These results are also comparable to values obtained by Schrijver et al. (1995) and Schmitt et al. (1996) and to values for the Sun (Drake et al. 2000).

\section{Conclusions}

The RGS and LETGS spectra of the corona of Procyon below $40 \AA$ are dominated by the $\mathrm{H}$ - and He-like transitions of $\mathrm{C}, \mathrm{N}$, and $\mathrm{O}$ and by Fe XVII lines. Above $40 \AA$

\footnotetext{
${ }^{4}$ Recent calculations for Fe VIII by Griffin et al. (2000) give for our observed Fe VIII ratio 131/168 = 0.14 a temperature of $\sim 2.5 \mathrm{MK}$, near the high-energy limit of the DEM distribution.
} 


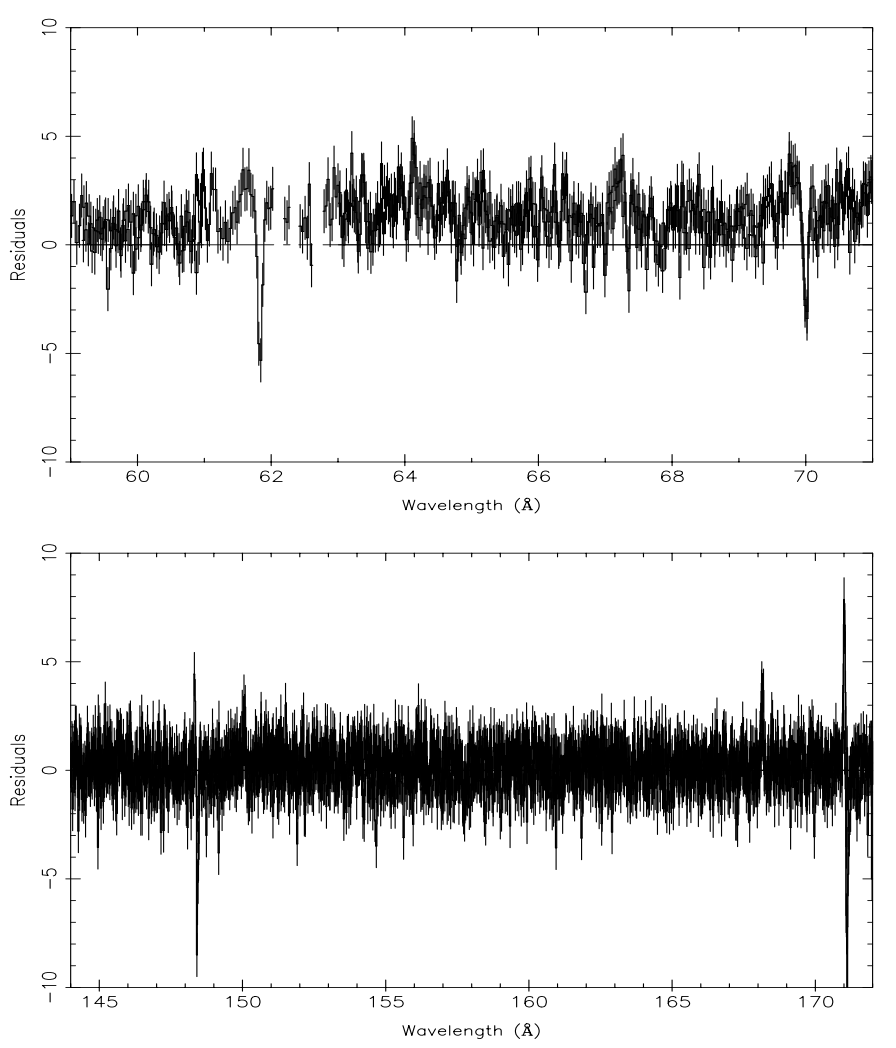

Fig. 4. Fit residuals ((observed - model)/error) of parts of the LETGS spectrum.

the LETGS spectrum shows many L-shell lines of e.g., Ne, $\mathrm{Mg}$, and $\mathrm{Si}$, together with lines of Fe VIII-XIII of which the Fe IX line at 171.075 is very prominent. All methods applied in Sect. 3.2 to the spectra of the RGS+MOS and the LETGS show temperatures of the corona of Procyon between 1-3 MK. No indication for a considerably higher temperature component $(T \gtrsim 4 \mathrm{MK})$ is found. The total EM obtained using RGS and LETGS is about $4.1(.5) \times 10^{50} \mathrm{~cm}^{-3}$. The $E M$ distribution shows a smooth continuous structure without separated peak structures. Our results improve on those of Schmitt et al. (1996) who obtain an $E M$ distribution with a maximum temperature around 1.6 MK and a cutoff beyond 6.3 MK. No significant variability of the coronal conditions took place between the observations by RGS and LETGS.

The abundances of $\mathrm{C}$ and $\mathrm{N}$, relative to $\mathrm{O}$ are somewhat higher ( factor 1.5) than the values obtained in the solar photosphere (Anders \& Grevesse 1989). The Fe abundance is about $1-1.5 \times$ solar. No significance for a FIP effect, as observed in the solar corona (Feldman et al. 1992), is found. The same was concluded by Drake et al. (1995), based on EUVE observations. This result is an exception of the trends found by Audard et al. (2001c) for RS CVn systems and by Güdel et al. (2001c) for solar analogs. These authors have found indications for the evolution from an inverse FIP effect for highly active stars - via the absence of a FIP effect in intermediately active stars - towards a normal FIP effect for less active stars.
Table 5. Observed line fluxes and fluxes obtained from the emissivity from the model.

\begin{tabular}{|c|c|c|c|c|}
\hline \multirow{2}{*}{$\begin{array}{c}\text { LETGS } \\
\text { Observed }\end{array}$} & \multicolumn{4}{|c|}{ Line identifications } \\
\hline & \multicolumn{4}{|c|}{ Model } \\
\hline$\lambda(\AA) \quad$ flux $^{a}$ & $\lambda(\AA)$ & 3 -T flux ${ }^{b}$ & DEM-flux $^{b}$ & Ion \\
\hline $18.9721 .83(15)$ & 18.973 & 1.93 & 1.80 & N VII \\
\hline $21.5973 .01(25)$ & 21.602 & 3.35 & 2.94 & N VII \\
\hline $24.7900 .80(14)$ & 24.781 & 0.76 & 0.70 & N VII \\
\hline $33.7314 .02(32)$ & 33.736 & 4.64 & 4.19 & C VI \\
\hline $40.2632 .29(36)$ & 40.270 & 2.03 & 1.40 & $\mathrm{C} \mathrm{V}$ \\
\hline $40.7181 .88(42)$ & 40.730 & 1.33 & & $\mathrm{C} \mathrm{V}$ \\
\hline \multirow[t]{2}{*}{$41.4751 .07(29)$} & 41.470 & 0.68 & & $\mathrm{C} \mathrm{V}$ \\
\hline & 41.480 & 0.28 & 0.31 & Ar IX \\
\hline $43.7430 .54(8)$ & 43.740 & 0.54 & 0.94 & Si XI \\
\hline $44.150 \quad 0.67(10)$ & 44.165 & 0.64 & 0.66 & Si XII \\
\hline $47.2420 .46(8)$ & 47.280 & 0.19 & 0.17 & $\operatorname{Mg} X$ \\
\hline $47.4520 .48(8)$ & 47.500 & 0.61 & 0.60 & S IX \\
\hline $47.6420 .49(8)$ & 47.654 & 0.20 & 0.31 & S X \\
\hline \multirow[t]{2}{*}{$49.2071 .44(14)$} & 49.220 & 0.43 & 0.95 & Si XI \\
\hline & 49.180 & 0.41 & 0.37 & Ar IX \\
\hline $50.5201 .68(15)$ & 50.530 & 1.48 & 1.55 & Si X \\
\hline $50.6861 .30(14)$ & 50.690 & 1.50 & 1.58 & Si X \\
\hline $52.3060 .75(11)$ & 52.300 & 0.45 & 0.74 & Si XI \\
\hline $61.0201 .41(25)$ & 61.050 & 2.51 & 1.98 & Si VIII \\
\hline $61.0871 .38(24)$ & & & & Si VIII ${ }^{c}$ \\
\hline $63.2830 .94(15)$ & 63.294 & 1.07 & 0.93 & $\operatorname{Mg} X$ \\
\hline \multirow[t]{2}{*}{$69.6462 .03(21)$} & 69.658 & 0.85 & 0.67 & Si VIII \\
\hline & 69.660 & 1.14 & 1.11 & Fe XV \\
\hline \multirow[t]{2}{*}{$74.8601 .10(18)$} & 74.854 & 0.51 & 0.57 & Mg VIII \\
\hline & 74.845 & 0.26 & 0.40 & Fe XIII \\
\hline $75.0351 .05(18)$ & 75.034 & 0.52 & 0.57 & Mg VIII \\
\hline $77.7401 .11(18)$ & 77.741 & 0.42 & 0.37 & Mg IX \\
\hline $86.7651 .13(17)$ & 86.772 & 0.71 & 0.45 & Fe XI \\
\hline $88.0871 .68(20)$ & 88.092 & 2.33 & 1.62 & Ne VIII \\
\hline $98.2512 .89(34)$ & 98.260 & 3.02 & 2.37 & Ne VIII \\
\hline $105.201 .22(21)$ & 105.21 & 0.32 & 0.19 & Fe IX \\
\hline $130.921 .78(39)$ & 130.94 & 0.29 & 0.20 & Fe VIII \\
\hline $131.211 .78(35)$ & 131.24 & 0.41 & 0.29 & Fe VIII \\
\hline $148.3611 .3(10)$ & 148.40 & 11.0 & 15.5 & $\mathrm{Ni} \mathrm{XI}$ \\
\hline $150.085 .08(60)$ & 150.10 & 2.8 & 2.63 & $\mathrm{O} \mathrm{VI}$ \\
\hline $152.115 .60(66)$ & 152.15 & 2.9 & 6.0 & Ni XII \\
\hline $167.433 .9(10)$ & 167.49 & 3.9 & 2.64 & Fe VIII \\
\hline $167.593 .9(12)$ & 167.66 & 4.0 & 2.74 & Fe VIII \\
\hline $168.137 .4(13)$ & 168.17 & 0.3 & 0.20 & Fe VIII \\
\hline $168.515 .4(13)$ & 168.54 & 2.0 & 1.42 & Fe VIII \\
\hline $168.905 .0(18)$ & 168.93 & 1.3 & 0.71 & Fe VIII \\
\hline $171.04114(8)$ & 171.08 & 100 & 78 & Fe IX \\
\hline
\end{tabular}

${ }^{a}$ Observed flux in $10^{-4}$ photons $/ \mathrm{cm}^{2} / \mathrm{s}$ with in parentheses $1 \sigma$ uncertainty in the last digits.

${ }^{b}$ Model flux in $10^{-4}$ photons $/ \mathrm{cm}^{2} / \mathrm{s}$.

${ }^{c}$ Sum of two Si VIII lines to be compared with model flux.

Clearly, the weakly active star Procyon does not fit into this picture.

Acknowledgements. The Space Research Organization Netherlands (SRON) is supported financially by NWO. The PSI group acknowledges support from the Swiss National Science Foundation (grant 2100-049343). We are grateful to 
the calibration teams of the instruments on board XMMNewton and Chandra. We thank Nancy Brickhouse and Jeremy Drake for their efforts to obtain a long LETGS observation. Finally, we are grateful to the referee for helpful comments.

\section{References}

Anders, E., \& Grevesse, N. 1989, Geochim. Cosmochim. Acta, 53, 197

Audard, M., Behar, E., Güdel, M., et al. 2001a, A\&A, 365, L329

Audard, M., Güdel, M., \& Mewe, R. 2001b, A\&A, 365, L318

Audard, M., Güdel, M., Sres, A., et al. 2001c, in Stellar Coronae in the Chandra and XMM-Newton Era, ed. F. Favata, \& J. J. Drake, ASP Conf. Ser.

Behar, E., Cottam, J., \& Kahn, S. M. 2001, ApJ, 548, 966

Beiersdorfer, P., Lepson, J. K., Brown, G. V., et al. 1999, ApJ, 519, L185

Bowyer, S., Drake, J. J., \& Vennes, S. 2000, ARA\&A, 38, 231

Brinkman, A. C., Behar E., Güdel, M., et al. 2001, A\&A, 365, L324

Brinkman, A. C., Gunsing, C. J. T., Kaastra, J. S., et al. 2000, ApJ, 530, L111

Dere, K. P., Landi, E., Mason, H. E., et al. 1997, A\&AS, 125, 149

Doschek, G. A., \& Cowan, R. D. 1984, ApJS, 56, 67

Drake, J. J., Laming, J. M., \& Widing, K. G. 1995, ApJ, 443, 393

Drake, J. J., Peres, G., Orlando, G., et al. 2000, ApJ, 545, 1074

Feldman, U., Mandelbaum, P., Seely, J. F., et al. 1992, ApJS, 81,387

Grevesse, N., \& Sauval, A. J. 1998, Space Sci. Rev., 85. 161

Griffin, D. C., Pindzola, M. S., \& Badnell, N. R. 2000, A\&AS, 142,317

Güdel, M., Audard, M., Briggs, K., et al. 2001a, A\&A, 365, L336

Güdel, M., Audard, M., Magee, H., et al. 2001b, A\&A, 365, L344

Güdel, M., Audard, M., Mewe, R., et al. 2001c, in Stellar Coronae in the Chandra and XMM-Newton Era, ed. F. Favata, \& J. J. Drake, ASP Conf. Ser.
Güdel, M., Guinan, E. F., \& Skinner, S. L. 1997, ApJ, 483, 947 den Herder, J. W., Brinkman, A. C., Kahn, S. M., et al. 2001, A\&A, 365, L7

Irwin, A. W., Fletcher, J. M., Yang, S. L. S., et al. 1992, PASP, 104,489

Kaastra, J. S., Mewe, R., Liedahl, D. A., et al. 1996b, A\&A, 314,547

Kaastra, J. S., Mewe, R., \& Nieuwenhuijzen, H. 1996a, in UV and X-ray Spectroscopy of Astrophysical and Laboratory Plasmas, ed. K. Yamashita, \& T. Watanabe (Universal Academy Press, Inc., Tokyo), 411 (SPEX)

Kelly, R. L. 1987, Atomic and Ionic Spectrum lines below 2000 Angstroms: Hydrogen through Krypton, J. Phys. Chem. Ref. Data 16, suppl. 1

Lepson, J. K., Beiersdorfer, P., Brown, G. V., et al. 2000, Rev. Mex. Astron. Astrofis. (Ser. Conf.), 9, 137

Lepson, J. K., Beiersdorfer, P., Brown, G. V., et al. 2002, ApJ, submitted

Linsky, J. L., Diplas, A., Wood, B. E., et al. 1995, ApJ, 451, 335

Meer, R. L. J., van der, et al. 2002, in preparation

Mewe, R., Gronenschild, E. H. B. M., \& van den Oord, G. H. J. 1985, A\&AS, 62, 197

Mewe, R., Kaastra, J. S., \& Liedahl, D. A. 1995, Legacy, 6, 16 (MEKAL)

Mewe, R., Raassen, A. J. J., Drake, J. J., et al. 2001, A\&A, 368,888

Ness, J.-U., Mewe, R., Schmitt, J. H. M. M., et al. 2001, A\&A, 367,282

Phillips, K. J. H., Mewe, R., Harra-Murnion, L. K., et al. 1999, A\&AS, 138, 381

Porquet, D., Mewe, R., Dubau, J., et al. 2001, A\&A, 376, 1113

Prieto, C. A., Lambert, D. L., \& Asplund, M. 2001, ApJ, 556, L63

Schmitt, J. H. M. M., Drake, J. J., Haisch, B. M., et al. 1996, ApJ, 467, 841

Schrijver, J., Mewe, R., van den Oord, G. H. J., et al. 1995, A\&A, 302, 438

Turner, M. J. L., Abbey, A., Arnaud, M., et al. 2001, A\&A, 365, L27

Wang, J.-S., Datla, R. U., \& Griem, H. R. 1984, Phys. Rev. A, 29,1558 